\title{
11 University
}

Moulin, H. (2016) Entropy, desegregation, and proportional rationing.

Journal of Economic Theory, 162, pp. 1-20. (doi:10.1016/j.jet.2015.12.002)

This is the author's final accepted version.

There may be differences between this version and the published version.

You are advised to consult the publisher's version if you wish to cite from it.

http://eprints.gla.ac.uk/116506/

Deposited on: 18 February 2016

Enlighten - Research publications by members of the University of Glasgow http://eprints.gla.ac.uk 


\title{
Entropy, Desegregation, and Proportional Rationing
}

\author{
Herve Moulin* \\ University of Glasgow
}

Revised, September 2015

\begin{abstract}
An assignment of students to schools displays zero-segregation if all schools have the same distribution of the different types (ethnic, geographical, gender, ..) of students. We axiomatize the choice of an optimally desegregated assignment under arbitrary capacity constraints. The celebrated Consistency axiom, together with standard rational choice requirements, identify the choice rule minimizing a canonical index of proportional fairness: the entropy of the assignment matrix. This is an alternative vindication of the Mutual Information index of segregation $([20])$, related to its characterization in [11].

A similar result holds in the capacity-constrained extension of the bipartite rationing model ([17]): there we must minimize the entropy of the rationing matrix augmented by individual deficits.

Keywords: assignment, bipartite rationing, proportionality, segregation, consistency, entropy

Acknowledgments: comments by Gabrielle Demange, Francois Maniquet, Jay Sethuraman, Oscar Volij, Peyton Young, and seminar participants in Glasgow, Oxford, Vienna, Cambridge, and Istanbul have been very helpful. Special thanks to the Associate Editor and two referees for their detailed remarks.

*herve.moulin@glasgow.ac.uk, phone:(44)1413302004, fax:(44)1413304939
\end{abstract}

\section{Introduction}

\subsection{Proportional assignment and rationing}

We consider two related resource allocation models. In the proportional assignment problem balls of different colors must be placed in bins of different sizes, and the total number of balls equals the total capacity of the bins. The goal is to achieve a representation of the colors in each bin as equal as 
possible across bins. The ideal assignment is proportional: the proportions of the different colors are the same in each bin. However we impose exogenous capacity constraints on the entries of the realized assignment: the number of color $a$ balls in bin $i$ is bounded from above and below. How to best approximate the ideal proportional assignment under such constraints?

The proportional rationing problem asks the same question but assumes that the total number of balls is smaller than the total capacity of the bins.

We avoid rounding problems by setting all variables as real numbers, and we characterize in both models a unique approximation rule that minimizes the entropy of the realized assignment (or a variant of this entropy in the rationing model) under the capacity constraints.

These results apply to, and connect, two disjoint literatures respectively on the measurement of segregation and on the rationing problem, a benchmark fair division model. We discuss these connections in turn.

Reducing segregation is an important issue of public policy (see e. g., [10]). Two paramount examples are the assignment of students of various types (ethinicity, gender, academic status, etc..) in th public schools of a certain district; and the distribution of jobs of different social status by gender, ethnicity, or in socio-economic groups more finely tuned by education, health, and so on. The zero-segregation ideal is proportional: in any two schools, or job types, the proportions of the different types are identical. Examples of exogenous capacity constraints include: there are not enough women qualified for a certain type of jobs; each school must enroll a certain amount of earmarked students; busing students is limited by geographic constraints; and so on.

An segregation index assigns zero to a perfectly proportional assignment, and only then; it increases to 1 or $\infty$ for maximally segregated assignments (e.g., a single type of student per school). Two good surveys are [13] and [11]. Choosing such an index provides an answer to our approximation challenge: given the capacity constraints, pick the feasible assignment with the smallest index. This is well defined if the index is a strictly convex function of the assignment matrices (once we fix the number of balls of each color and the size of each bin), which is the case for many indices discussed in the literature: the Dissimilarity and Gini indices, the family of Atkinson's indices, and the Mutual Information index.

Our proportional assignment model approaches the desegregation problem from the choice function perspective: instead of deciding which of any two assignment matrices is more segregated, we select one such matrix for any capacity-constrained problem. We impose two familiar rationality properties (explained in the next subsection) on this choice function, and characterize the rule choosing the feasible matrix minimizing the Mutual Information 
index due to Theil ([20]) and axiomatized by Frankel and Volij ([11]). We stress that our result is not a proper characterization of this index because the choice sets have the particular rectangular shape implied by the capacity contraints.

In the classic rationing problem (see e. g., Chapter 2 in [15]), balls of a single color (the resource) are distributed to bins, each with its own capacity (demand), and total capacity exceeds the number of balls (there is excess demand of balls). A rationing rule is a systematic way to distribute the resource, i.e., an interpretation of distributive justice in this simple model. The proportional rule is by far the oldest and most natural interpretation (more on this in section 2).

Here we introduces the bipartite version with capacity constraints of the rationing problem (it generalizes the model of [17], [18], see Section 2) and we look for the right interpretation of "proportional fairness" with the same choice approach. Examples include the distribution of goods of different quality to the retail stores of the chain, of jobs of different difficulty to company workers, of customers of different types to salespersons, etc.. Fairness demands that every store/worker/salesperson gets the same proportion of high, medium and low quality goods/jobs/customers. Capacity constraints capture the fact that some stores can only keep so much of certain goods; that some workers are not qualified for certain jobs, or need a minimal amount of some jobs for training purposes, and so on. The same two rationality properties on the choice function single out the rule minimizing the total entropy of the assignment matrix, plus that of the deficits in each bin.

The next subsection explains both rules, and the axioms we use to characterize them.

\subsection{Capacited proportionality and the choice axioms}

We replace the integer-valued balls and bins terminology by the more general network terminology of sources and sinks. The finite set of sources is $A$ and $r_{a}$ is the total capacity of source $a \in A$; and a finite set $N$ of sinks and sink $i \in N$ has a total capacity $x_{i}$. In the assignment model, the balancedness equality $x_{N}=r_{A}$ holds $^{1}$, while in the rationing model we have $x_{N} \geq r_{A}$. In both models we must choose a non negative matrix $\left[y_{i a}\right]$, shipping the total source capacity to the sinks: $y_{N a}=r_{a}$ for all $a$ and $y_{i A} \leq x_{i}$ for all $i$. The proportional matrix $y_{i a}=\frac{x_{i} r_{a}}{x_{N}}$ must be chosen when it is feasible.

The capacity constraints apply to each entry $y_{i a}$ of $y: 0 \leq q_{i a}^{-} \leq y_{i a} \leq$ $q_{i a}^{+} \leq+\infty$. Let $E(z)=z \ln (z)$ be the entropy function. If $x_{N}=r_{A}$ the

\footnotetext{
${ }^{1}$ Notation $x_{N}=\sum_{N} x_{i}, y_{N a}=\sum_{N} y_{i a}$ etc.
} 
capacited proportional assignment $y$ minimizes total entropy $\sum_{N \times A} E n\left(y_{i a}\right)$ under these constraints. If $x_{N} \geq r_{A}$ the capacited proportional rationing minimizes $\sum_{N \times A} E n\left(y_{i a}\right)+\sum_{N} E n\left(x_{i}-y_{i A}\right)$ : this is the entropy of $y$ plus that of the sink deficits $x_{i}-y_{i A}$.

Consider for comparison the Mutual Information index:

$$
M I(y)=\sum_{i, a} E n\left(y_{i a}\right)-\sum_{i} E n\left(y_{i A}\right)-\sum_{a} E n\left(y_{N a}\right)+E n\left(y_{N A}\right)
$$

In an assignment problem all terms $y_{i A}, y_{N a}$ and $y_{N A}$ are constant, therefore the capacited proportional matrix minimizes the MI index under the constraints. This is no longer true for the capacited proportional rationing matrix.

Our two key axioms are familiar rationality requirements.

The first one is the cornerstone of the rational choice approach: if an assignment/rationing matrix is optimal under certain constraints, and satisfies tighter constraints, it should still be optimal under the latter constraints; moreover a non binding constraint can be ignored. Both statements hold when the chosen matrix minimizes a strictly convex function under constraints. They are both compelling when the exogenous capacity constraints do not have any normative content and for this reason we call this requirement Constraint Neutrality.

The Consistency axiom means that if we fix the capacity constraints and the rule selects a certain matrix $y=\left[y_{i a}\right]$, then any submatrix of $y$ is still selected by the rule in the corresponding subproblem. This is a famous separability property: "every part of a correct outcome should be correct". 2

We characterize both the capacited proportional chice rule both in the assignment and rationing contexts, by the combination of Constraint Neutrality and Consistency as well as standard Symmetry and Continuity requirements. We stress that Constraint Neutrality and Consistency are both orthogonal to fairness, in the sense that they do not rule out very unfair rules. The only fairness requirements in our characterization are Symmetry and the fact that full proportionality must be chosen when feasible.

Although an assignment problem is the special case of a rationing problem where sources and sinks capacities are balanced, the rationing characterization does not imply the assignment one, because the axioms bear on two nested domains of problems. But our parallel discussions of assignment and rationing highlight their similarities.

\footnotetext{
${ }^{2}$ We modify Balinski and Young's: "every part of a fair division should be fair" ([2]) to stress, like Thomson ([23]), that CSY is satisfied by some very unfair rules.
} 


\subsection{Contents}

Section 2 reviews the relevant literature. Section 3 introduces our model and the most important axioms. The capacited proportional assignment and rationing rules are defined in Section 4, where we give an intuitive multiplicative parametrization of these matrices (Proposition 1 and Corollary 1), that plays a key role in the proofs and implies several natural monotonicity properties of our rules. Section 5 states the characterization result, Theorem 1 , and an open question. Section 6 contains most proofs.

\section{Related work}

On the Mutual Information index In addition to providing an excellent survey of the main segregation indices in the literature, Frankel and Volij ([11]) single out the Mutual Information index (1): they axiomatize the ordering of assignment matrices defined by this index (their Theorem 2). Statement $i$ ) in our Theorem is not an alternative characterization of this index because our assignment rules (Definition 2) choose from special sets of matrices with constant sums in rows and columns and a "rectangular" shape from the capacity constraints. ${ }^{3}$ Our rules do not allow comparisons of matrices with different sums in rows or columns, or different sizes. Nor do they tell us what to choose from a general convex set of matrices: see the open question in Section 5.

Despite these differences, Frankel and Volij's combination of axioms resembles ours. Both include Symmetry and Continuity requirements. Our Constraint Neutrality is implied by, but does not imply, the existence of an ordering of matrices that we minimize under neutral constraints, which Frankel and Volij assume. Our critical Consistency axiom is similar in spirit and in bite to their Independence axiom, requiring that if two assignment matrices differ only in a submatrix, what happens in the submatrix is all we need to compare the two matrices. Though Consistency and Independence are not logically related, Independence is the classic assumption implying a separably additive utility representation of the ordering, just like Consistency, in rationing problems and in their bipartite generalization, forces the minimization of such a utility (see below the discussion of bilateral assignment rules).

\footnotetext{
${ }^{3}$ For instance with 2 sources and two sinks, the set of feasible matrices is an interval in a line of $\mathbb{R}^{4}$. Then any strictly convex function on that line minimal at the proportional assignment is an index that our capacited proportional rule minimizes under any capacity constraints.
} 
Frankel and Volij need three additional requirements to capture the $\mathrm{Mu}-$ tual Information index. One is the familiar Scale Invariance. Next School Division says that when we merge the students of two schools into a single school, segregation can only decrease; and Group Division says that segregation does not change if we merge two ethnic groups with identical distributions across schools. We require no similar property in our model, where Consistency is about dropping, not merging, rows and columns.

Finally Frankel and Volij also characterize the Atkinson segregation index (their Theorem 1), about which we have nothing to say as it does not define a consistent choice function.

On rationing rules In the simple one-source version of the rationing model, we must divide the amount $r$ of resources between agents $i$ with demands $x_{i}$. Although many rules have been proposed and axiomatized (see the survey [21]), the proportional rule $y_{i}=\frac{x_{i} r}{x_{N}}$ stands out as the most popular. It appears in Aristotle's Nicomachean Ethics (see [9], [19] for a historical and legal discussion), and was the first rationing rule offered a modern axiomatic justification ([3]). It is the compelling rule to divide the estate of a bankrupt firm between creditors with equal status([14]).

Originally introduced for the related apportionment problem ([2]), Consistency has been at the forefront of the fair division literature for the last three decades (see [25], [22]). Young's Theorem ([24]) providing a parametric representation of consistent rationing rules is perhaps its most successful application.

The introduction of upper and lower capacity constraints in the onesource rationing model is not new. Bergantions and Sanchez ([5]) and Hougaard et al. ([12]) introduce such constraints as well, but give them normative content: for instance a lower bound represents a status quo ex ante, and gains are rescaled accordingly. This contradicts our Constraint Neutrality requirement, which is critical to capture rules minimizing some measure of segregation under constraints.

On bipartite rationing rules The model of bipartite rationing with multiple sources and sinks is first introduced by Bochet et al. ([6], [7]) in a context where agents are endowed with single-peaked preferences over their share and must report them truthfully. Then Moulin and Sethuraman ([17] and [18]) develop an axiomatic, incentive-free version of the same model, where capacity constraints are " $0 / \infty$ ": we have $q_{i a}^{-}=0$ for all $i a$ and $q_{i a}^{+}$is either 0 or $+\infty$; for instance certain workers are unable to do certain jobs. They discuss the consistent extension of several one-source rationing meth- 
ods: proportional, uniform gains and losses in [17], and the rich family of loss-calibrated methods in [18]. Theorem 1 in [17] is a characterization of capacited proportional rationing similar to statement $i i$ ) in Theorem 1 here. The Consistency requirement is weaker in that it applies to the removal of sources, not sinks; and Constraint Neutrality has no bite under $0 / \infty$ constraints. Note that this result is not a consequence of statement $i i$ ) in our Theorem 1 because it applies to a smaller domain of problems.

On the other hand our characterization of capacited proportional assignment (statement $i$ ) has no counterpart in the model with $0 / \infty$ capacities: indeed in that model it is an open question to understand the consequences of Consistency.

On bilateral assignment rules In the bilateral assignment model without capacity constraints, the companion paper [16] drops the assumption that proportionality is the first best and explores the consequences of Consistency (together with Symmetry and Continuity). Under additional monotonicity and limit requirements, it turns out that a consistent rule selects the assignment minimizing a separably additive function $\sum_{N \times A} W\left(y_{i a}\right)$ where $W$ is a smooth and strictly convex function. Then $W$ is the entropy function if (and only if) the proportional assignment is the first best. A similar result for the bipartite rationing model is described in Section 7 of [16].

In the special case where $|N|=|A|$ and $x_{i}=r_{a}=1$, an assignment $y=$ $\left[y_{i a}\right]$ is a doubly stochastic matrix and can be interpreted as a probabilistic assignment of rows to columns. In the unconstrained version of that model, Chambers ([8]) uses a stronger version of Consistency to capture the uniform assignment, a special case of the proportional one. Note that our version of Consistency cannot be applied to the domain of doubly stochastic matrices because it requires arbitrary sums in rows and columns.

On Fair Representation Apportioning seats in a parliament to voting districts in proportion to their population poses an interesting rounding problem. Balinski and Young in their classic book on apportionment ([2]) argue for a particular rounding method on the basis of the Consistency axiom itself (Uniformity in their terminology). In order to allocate seats to political parties and townships, Balinski and Demange ([1]) generalize the apportionment problem to two dimensions. Given an arbitrary exogenous assignment matrix $\left[z_{i a}\right]$, and capacity constraints on the sums of rows and of columns (but not on the entries of the matrix), they look for a feasible assignment $\left[y_{i a}\right]$ "as proportional as possible" to the initial matrix $z$. By contrast in our model we look for an assignment as close as possible to the ideal proportional 
matrix, given fixed sums in rows and columns and exogenous constraints on the entries of the matrix.

Allowing for real valued assignments, Balinski and Demange combine Consistency with Scale Invariance and Monotonicity (of $z \rightarrow y$ ), to derive a solution minimizing a weighted entropy similar to our capacited proportional assignment. Their parametric representation of the solution is a relative of our Proposition 1 in Section 4.

\section{Model}

The sets $N$ of sinks and $A$ of sources are both finite and non empty, with generic elements $i$ and $a$ respectively.

Notation. If $w \in \mathbb{R}_{+}^{N \times A}, S$ is a subset of $N$, and $T$ a subset of $A$, we write $w_{S a}=\sum_{i \in S} w_{i a}, w_{i T}=\sum_{a \in T} w_{i a}$, and $w_{S T}=\sum_{i \in S, a \in T} w_{i a}$. Finally we write $S^{c}=N \backslash S$ and $T^{c}=A \backslash T$ when this causes no confusion.

\subsection{Assignment and rationing problems}

An assignment or rationing problem $P=(N, A, x, r, Q)$ specifies

- the total capacity $x_{i}$ of each sink, so $x \in \mathbb{R}_{+}^{N}$

- the total capacity $r_{a}$ of each source a, so $r \in \mathbb{R}_{+}^{A}$

- for each $(i, a) \in N \times A$, a closed capacity interval $Q_{i a}=\left[q_{i a}^{-}, q_{i a}^{+}\right]$or $\left[q_{i a}^{-}, \infty\left[\subseteq \mathbb{R}_{+}\right.\right.$, so $Q=\Pi_{N \times A} Q_{i a} \subseteq \mathbb{R}_{+}^{N \times A}$

The budget balance equation $x_{N}=r_{A}=b$ holds in an assignment problem. In a rationing problem we have $x_{N} \geq r_{A}$.

If $Q_{i a}=\mathbb{R}_{+}$for all $(i, a) \in N \times A$, we speak of an unconstrained problem, and we write simply $P=(N, A, x, r)$.

A feasible assignment (resp. rationing) matrix is $y \in Q$ such that $y_{N a}=$ $r_{a}$ for all $a \in A$, and $y_{i A}=x_{i}$ (resp. $\left.y_{i A} \leq x_{i}\right)$ for all $i \in N$. Such a matrix always exists if $P$ is unconstrained, but in general its existence is not guaranteed.

Lemma 1 The assignment problem $P=(N, A, x, r, Q)$ is feasible, i.e., there is at least one feasible matrix $y$, if and only if for all $S, \varnothing \subseteq S \subseteq N$, and $T, \varnothing \subseteq T \subseteq A$, not both empty, we have

$$
r_{T}+q_{S T^{c}}^{-} \leq x_{S}+q_{S^{c} T}^{+}
$$


The rationing problem $P=(N, A, x, r, Q)$ is feasible if and only if (2) holds, and in addition

$$
q_{N a}^{-} \leq r_{a} \text { for all } a \in A
$$

Proof That (2) and (3) are necesary for feasibility is obvious. Note that if $x_{N}=r_{A}$ and $P$ is feasible, inequality (3) follows from (2) by taking $T=A \backslash\{a\}$ and $S=N$.

To check that (2) is sufficient when $x_{N}=r_{A}$, we consider the flow graph with capacity constraints $Q_{i a}$ on each edge from source $a$ to sink $i$, one additional source $\alpha$ connected to each source $a$ by an edge with capacity $r_{a}$, and one additional sink $\beta$ connected to each sink $i$ by an edge with capacity $x_{i}$. We must show the maxflow is $r_{A}$ : by the max-flow min-cut theorem this amounts to show that each cut has a capacity at least $r_{A}$. Fix such a cut $C \cup\{\beta\}$ where $\varnothing \subseteq C \subseteq N \cup A$, and define $S, T$ as follows

$$
C \cap N=S^{c} ; C \cap A=T^{c}
$$

The capacity of $C$ is $\gamma=x_{S}+q_{S^{c} T}^{+}-q_{S T^{c}}^{-}+r_{T^{c}}$, therefore $\gamma \geq r_{A}$ is precisely inequality (2) (if both $S, T$ are empty then the capacity of $C$ is exactly $r_{A}$ ).

If $x_{N} \geq r_{A}$ it is enough to show there exists some $x^{\prime} \in \mathbb{R}_{+}^{N}$ such that $x_{N}^{\prime}=r_{A}, x^{\prime} \leq x$, and $x^{\prime}$ meets (2) for all $S, T$. The latter means

$$
x_{S}^{\prime} \geq v(S)=\max _{T \subseteq A}\left\{r_{T}+q_{S T^{c}}^{-}-q_{S^{c} T}^{+}\right\} \text {for all } S \varsubsetneqq N
$$

and (3) implies $r_{A}=\max _{T \subseteq A}\left\{r_{T}+q_{N T^{c}}^{-}\right\}=v(N)$. Thus $x^{\prime}$ must be in the core of the TU game $(N, v)$, and below $x$. Now $(N, v)$ is clearly convex, therefore such an $x^{\prime}$ exists if ( and only if) $x_{S} \geq v(S)$ for all $S$ including $N$, which (2) and (3) guarantee.

We write $\mathcal{A}$ and $\mathcal{R}$ respectively for the set of feasible assignment and rationing problems, and $\Phi(P)$ for the set of feasible matrices in the feasible problem $P$. Then $\mathcal{A}^{u} \subset \mathcal{A}$ and $\mathcal{R}^{u} \subset \mathcal{R}$ are the subsets of unconstrained problems.

Before starting with the definition and axiomatization of choice rules, we must address an important technical issue, namely the fact that the capacity constraints may determine several entries $y_{i a}$ of the (assignment or rationing) matrix, beyond those such that $q_{i a}^{-}=q_{i a}^{+}$. Here is an assignment example with three sources $a, b, c$, three sinks $A, B, C$, and four lower or upper bounds (there are no other capacity constraints):

$$
\begin{array}{r}
\left|\begin{array}{l}
x_{A}=2 \\
x_{B}=4 \\
x_{C}=6
\end{array}\right|\left|\begin{array}{ccc}
\cdot & \cdot & y_{A c} \geq 0 \\
\cdot & \cdot & y_{B c} \geq 2 \\
y_{C a} \leq 3 & y_{C b} \leq 1 & \cdot
\end{array}\right| \\
\left|\begin{array}{lll}
r_{a}=4 & r_{b}=4 & r_{c}=4
\end{array}\right|
\end{array}
$$


Together the sources $a, b$ with capacity 8 send at most 4 to sink $C$, hence at least 4 to the two sinks $A, B$; those two sinks get at least 2 from source $c$ : thus sinks $A, B$ get exactly 4 from $a, b$ and exactly 2 from $c$. In turn each entry in $\{C\} \times\{a, b\}$ and $\{A, B\} \times\{c\}$ is determined:

$$
\left|\begin{array}{ccc}
\left(0 \leq y_{A a} \leq 1\right) & \left(1 \leq y_{A b} \leq 2\right) & y_{A c}=0 \\
\left(0 \leq y_{B a} \leq 1\right) & \left(1 \leq y_{B b} \leq 2\right) & y_{B c}=2 \\
y_{C a}=3 & y_{C b}=1 & \left(y_{C c}=2\right)
\end{array}\right|
$$

We fix a rationing problem $P \in \mathcal{R}$ and we let $\tau(P)$ be the set of all entries $i$ a such that $y_{i a}$ is constant in $\Phi(P)$, and $\sigma(P)$ be the set of those $i a$ such that $\left|Q_{i a}\right|=1$, i.e., $q_{i a}^{-}=q_{i a}^{+}$. Obviously $\sigma(P) \subseteq \tau(P)$, and as shown by the example above the inclusion can be strict. Suppose that for some non empty subsets $S \subseteq N$ and $T \subseteq A$, inclusions not both equalities, inequality (2) is in fact an equality: $r_{T}+q_{S T^{c}}^{-}=x_{S}+q_{S^{c} T}^{+}$. Combining this with the two accounting statements $r_{T}=y_{S T}+y_{S^{c} T}$ and $x_{S} \geq y_{S T}+y_{S T^{c}}$ gives

$$
y_{S^{c} T}+q_{S T^{c}}^{-} \geq y_{S T^{c}}+q_{S^{c} T}^{+}
$$

Equivalently

$$
\left(y_{S T^{c}}-q_{S T^{c}}^{-}\right)+\left(q_{S^{c} T}^{+}-y_{S^{c} T}\right) \leq 0
$$

which, together with $q^{-} \leq y \leq q^{+}$, implies $y=q^{-}$on $S \times T^{c}$ and $y=q^{+}$on $S^{c} \times T$.

It is critical in some proofs to restrict attention to problems where such implied constraints do not appear.

Definition 1 A problem $P$ in $\mathcal{A}$ is irreducible if (2) is a strict inequality when at least one of $S \varsubsetneqq N$ and $T \varsubsetneqq A$ holds. A problem $P$ in $\mathcal{R} \backslash \mathcal{A}$ is irreducible if, in addition, inequality (3) is strict for all a. We write $\mathcal{A}^{\text {ir }}$ and $\mathcal{R}^{i r}$ for the sets of irreducible assignment and rationing problems.

In subsection 6.1 of the Appendix we show that any feasible problem can be decomposed into independent irreducible problems: Lemmas 2,3. This canonical decomposition is important for our main proofs.

\subsection{Assignment and rationing rules}

We restrict attention to rules treating all sinks, and all sources symmetrically. We also require that a small change in the capacities $x_{i}, r_{a}$ has only a small influence on the solution.

If $\pi$ is a bijection of $N$, from the new label $i$ to the old label $\pi(i)$, and $y \in \Phi(P)$ is a matrix with the old labels, the same matrix with the new labels is $y^{\pi}: y_{i a}^{\pi}=y_{\pi(i) a}$. Define similarly $x^{\pi}, Q^{\pi}$, and $P^{\pi}=\left(N, A, x^{\pi}, r, Q^{\pi}\right)$. 
Definition $2 A$ assignment rule $F$ selects a feasible matrix $F(P)=y \in$ $\Phi(P)$ for every problem $P \in \mathcal{A}$. Moreover the mapping $F$ has the following properties:

- Symmetry in $N$ : for any $P \in \mathcal{A}$ and bijection $\pi$ of $N, F(P)^{\pi}=F\left(P^{\pi}\right)$

- Symmetry in $A$ : same property upon exchanging the roles of $N$ and A

- Continuity of the mapping $\mathcal{A} \ni(x, r, Q) \rightarrow F(P)$, for any fixed $N, A$

Upon replacing $\mathcal{A}$ by $\mathcal{R}$ the definition of a rationing rule $H$ is identical. We write $\mathcal{F}$ for the set of assignment rules, and $\mathcal{H}$ for that of rationing rules.

The next property, already discussed in the introduction, follows from our assumption that the constraints $Q$ are normatively neutral, they have no bearing on the fairness of the outcome.

We fix an assignment rule $F \in \mathcal{F}$ or a rationing rule $H \in \mathcal{H}$ :

- Constraint Neutrality (NEUT): fix any $P=(N, A, x, r, Q) \in \mathcal{A}$ and set $y=F(P) ; i)$ for any $Q^{\prime} \subset Q$, if $y \in Q^{\prime}$ then $y=F\left(P^{\prime}\right)$, where $P^{\prime}=\left(N, A, x, r, Q^{\prime}\right)$; ii) for any $i a \in N \times A$ such that $q_{i a}^{-}<y_{i a}$ (resp. $\left.y_{i a}<q_{i a}^{+}\right)$, then $y=F\left(P^{\prime}\right)$ where $Q_{i a}^{\prime}=\left[0, q_{i a}^{+}\right]$(resp. $\left[q_{i a}^{-}, \infty[)\right.$ and $P^{\prime}=P$ otherwise. The definition is identical for a rationing rule $H$.

Both statements express a version of "independence of irrelevant constraints", the first one with respect to a tightening of the constraints, the second one when we loosen a non binding constraint. Statement $i$ ) holds whenever $F(P)$ or $H(P)$ is the unique matrix minimizing over $\Phi(P)$ some numerical index (or ordinal ordering), possibly depending on $N, A, x$ and $r$, but not on $Q$. Statement $i i$ ) holds when the numerical index (or ordering) is convex over the set of feasible matrices for $(N, A, x, r)$, as is the case for the rules discussed in the next section.

The second key property is Consistency. Given a rationing rule $H \in \mathcal{H}$, a problem $P=(N, A, x, r, Q) \in \mathcal{R}$, the matrix $H(P)=y$, and two non empty subsets $S \subseteq N, T \subseteq A$, we define the subproblem $P[H ; S, T]=(S, T, \widetilde{x}, \widetilde{r}, Q)$ where $\widetilde{x}_{i}=x_{i}-y_{i T^{c}}, \widetilde{r}_{a}=r_{a}-y_{S^{c} a}$, and we abuse notation by still writing $Q$ for the constraints of the reduced problem (constraints outside $S \times T$ are now irrelevant).

- Consistency (CSY) in $\mathcal{H}$ : for any $P \in \mathcal{R}$ and non empty $S \subseteq$ $N, T \subseteq A, H(P[H ; S, T])$ is the projection of $H(P)$ on $S \times T$. 
For an assignment rule $F \in \mathcal{F}$, we can use exactly the same definition, or an alternative, perhaps more intuitive formulation going back to [2]. Given $N, A$, the constraints $Q$, and a matrix $y \in Q$, we say that this matrix is $F$-fair for $Q$ if the rule $F$ chooses $y$ in the problem $(N, A, x, r, Q)$ defined by $x_{i}=y_{i A}$ for all $i$, and $r_{a}=y_{N a}$ for all $a$.

- Consistency (CSY) in $\mathcal{F}$ : every submatrix of a matrix $F$-fair for $Q$ is $F$-fair for the restriction of $Q$.

Note that CSY in $\mathcal{H}$ has no bite if $|N|=1$, and CSY in $\mathcal{F}$ has no bite if $|N|=1$ and/or $|A|=1$, because $\Phi(P)$ is then a singleton.

Here is a simple consequence of CSY: if $x_{i}=0$ for some $i$, then $y_{i a}=0$ for all $a$, so the submatrix obtained after deleting row $i$ has all the same sums in rows and columns, and CSY allows us to simply delete $i$ altogether; similarly if $r_{a}=0$. Thus we can always assume when convenient $x, r \gg 0$.

A simple example with three sinks and a single source gives some intuition for the Consistency property. We share 40 units between the sinks with respective capacities $x_{1}=21, x_{2}=15, x_{3}=24$. In the absence of constraints, proportional division gives $y_{1}=14, y_{2}=10, y_{3}=16$. When we add the constraints $y_{1} \leq 13$ and $y_{3} \geq 20$, it is natural to propose the allocation $y_{1}^{\prime}=13, y_{2}^{\prime}=7, y_{3}^{\prime}=20$, where the shares of agents 1 and 3 suffer the smallest feasible correction. However this is not consistent: in the subproblem between agents 1 and 2, they share 20 units as 13 and 7 respectively, while the division $\widetilde{y}_{1}=11 \frac{2}{3}, \widetilde{y}_{2}=8 \frac{1}{3}$ is feasible and proportional to their demands 21 and 15 . Our rule recommends precisely $\widetilde{y}_{1}=11 \frac{2}{3}, \widetilde{y}_{2}=8 \frac{1}{3}, \widetilde{y}_{3}=20$.

\section{Capacited proportional assignment and ra- tioning}

In an unconstrained assignment problem $P \in \mathcal{A}^{u}$, the proportional flow $y_{i a}=$ $\frac{x_{i} r_{a}}{b}$ (recall $b=x_{N}=r_{A}$ ) is the unique minimum of a great variety of functions $\mathcal{W}$ strictly convex over $\Phi(P)$. Two examples are the Atkinson segregation index $\mathcal{W}(y)=1-\sum_{N} \Pi_{A}\left(\frac{y_{i a}}{r_{a}}\right)^{\frac{1}{|A|}}$, and its dual $\mathcal{W}(y)=1-\sum_{A} \Pi_{N}\left(\frac{y_{i a}}{x_{i}}\right)^{\frac{1}{|N|}}$. By minimizing $\mathcal{W}$ over $\Phi(P)$ for any $P \in \mathcal{A}$, we obtain a assignment rule $F \in \mathcal{F}$ : Symmetry is clear, and Continuity follows from Berge's Theorem. Constraint Neutrality is equally clear. ${ }^{4}$ But only one rule constructed in this

\footnotetext{
${ }^{4}$ We can also minimize some convex, but not strictly convex, functions $\mathcal{W}$ derived from other segregation indices such as the Dissimilarity index $\mathcal{W}(y)=\sum_{N \times A}\left|y_{i a}-\frac{x_{i} r_{a}}{b}\right|$, and the Gini index $\mathcal{W}(y)=\sum_{A \times N \times N} x_{i} x_{j}\left|\frac{y_{i a}}{x_{i}}-\frac{y_{j a}}{x_{j}}\right|:$ the proportional flow is still the unique minimum in an unconstrained problem, but in a constrained problem we need to deal with possibly multiple minima.
} 
way meets Consistency, the one that minimizes the (negative of the) entropy of the assignment matrix.

For unconstrained rationing problems $P \in \mathcal{R}^{u}$, we select similarly the proportional rationing $y_{i a}=\frac{x_{i} r_{a}}{x_{N}}$ by minimizing many strictly convex functions $\mathcal{W}$, for instance $\mathcal{W}(y)=\sum_{N \times A} \frac{y_{i a}^{\alpha}}{\left(x_{i} r_{a}\right)^{\alpha-1}}$, for any parameter $\alpha>1 .^{5}$ This yields as above a rationing rule $H \in \mathcal{H}$. Again, Consistency is not warranted, except for the choice of $\mathcal{W}$ in statement ii) below.

We recall the notation $E n(z)=z \ln (z)$.

\section{Definition 3}

i) The capacited proportional assignment rule $F^{\text {en }}$ selects for all $P \in \mathcal{A}$ the unique solution of the following program:

$$
\min _{y \in \Phi(P)} \sum_{N \times A} \operatorname{En}\left(y_{i a}\right)
$$

ii) The capacited proportional rationing rule $H^{\text {en }}$ selects for all $P \in \mathcal{R}$ the unique solution of the following program:

$$
\min _{y \in \Phi(P)} \sum_{N \times A} \operatorname{En}\left(y_{i a}\right)+\sum_{N} \operatorname{En}\left(x_{i}-y_{i A}\right)
$$

The two programs minimize a strictly convex function, thus $F^{e n}$ and $H^{e n}$ meet Definition 2 and Constraint Neutrality. To check Consistency of $F^{e n}$ we fix $N, A$, a set of constraints $Q$, and an assignment matrix $y$. The latter is fair for $Q$ if it minimizes $\sum_{N \times A} E n\left(y_{i a}\right)$ when we freeze all the sums $y_{i A}$ and $y_{N a}$. If $\widetilde{y}$ is a submatrix $S \times T$ of $y$, the corresponding sum $\sum_{S \times T} E n\left(y_{i a}\right)$ is a subset of the larger sum, thus $\widetilde{y}$ is still fair. ${ }^{6}$ Checking that $H^{e n}$ meets CSY is equally easy, because in the subproblem $P[H ; S, T]$ we have $\widetilde{x}_{i}-y_{i T}=x_{i}-y_{i A}$.

We check that the rules $F^{e n}, H^{e n}$, select the proportional assignment when there are no constraints. In an unconstrained rationing problem $P \in \mathcal{R}^{u}$ such that $x_{N}>r_{A}$ and $x, r \gg 0$, the KKT (Karoush Kuhn Tucker) conditions $\ln \left(y_{i a}\right)-\ln \left(x_{i}-y_{i A}\right)=\ln \left(y_{j a}\right)-\ln \left(x_{j}-y_{j A}\right)$ are clearly satisfied by the proportional matrix, and by this matrix only. If $P$ is balanced (so $P \in \mathcal{A}^{u}$ ) and $x, r \gg 0$, we can argue by Continuity that the solution of (4) is still proportional, or we can check directly that the solution of (4) has $y_{i a}>$ 0 because $(E n)^{\prime}(0)=-\infty$, then observe that the KKT conditions imply $\ln \left(y_{i a}\right)+\ln \left(y_{j b}\right)=\ln \left(y_{i b}\right)+\ln \left(y_{j a}\right) \Longleftrightarrow \frac{y_{i a}}{y_{j a}}=\frac{y_{i b}}{y_{j b}}$.

\footnotetext{
${ }^{5}$ If $x_{N}>r_{A}$, the KKT conditions $\left(\frac{y_{i a}}{x_{i} r_{a}}\right)^{\alpha-1}=\left(\frac{y_{j a}}{x_{j} r_{a}}\right)^{\alpha-1}$ are satisfied by the (interior) proportional matrix. By Continuity the minimum of $\mathcal{W}$ is still proportional when $x_{N}=r_{A}$.

${ }^{6}$ For the other functions $\mathcal{W}(y)$ mentioned above, the minimization program solved by $\widetilde{y}$ is not comparable to that solved by $y$ because the parameters $x_{i}, r_{a}$, have changed.
} 
Relation to the Mutual Information index Given an assignment problem $P \in \mathcal{A}$, all column and row sums $y_{N a}$ and $y_{i A}$, as well as $y_{N A}$, are the same for any $y \in \Phi(P)$. Therefore program (4) coincides with the minimization of the Mutual Information index $M I(y)((1))$ over $\Phi(P)$. In this sense our Theorem in Section 5 is an additional justification of this index, different to its axiomatic characterization by Frankel and Volij ([11]).

However in a rationing problem $P \in \mathcal{R}$, minimizing $M I(y)$ over $\Phi(P)$ and program (5) are two different things. In $\Phi(P)$ only the terms $y_{N a}$ and $y_{N A}$ are fixed, so minimizing $M I$ amounts to minimize $\sum_{N \times A} \operatorname{En}\left(y_{i a}\right)-\sum_{N} \operatorname{En}\left(y_{i A}\right)$ : for instance if $P \in \mathcal{R}^{u}$ and the assignment $y_{i a}=\frac{r_{a}}{|N|}$ is feasible, it minimizes $M I$, but $H^{e n}(P)$ chooses $y_{i a}=\frac{x_{i} r_{a}}{x_{N}}$.

Multiplicative parametrization of $F^{e n}$ and $H^{e n} \quad$ We give a multiplicative parametrization of the matrices $F^{e n}(P)$ and $H^{e n}(P)$, critical to the axiomatic characterization of these rules in the next section. It also gives an intuitive understanding of these rules and their monotonicity properties. The simplest statement applies only irreducible matrices.

Given any three real numbers we write $u *[v, w]$ for the projection of $u$ on the interval $[v, w]$, i.e., the median of $u, v, w$.

\section{Proposition 1}

i) Assignment: for any $P \in \mathcal{A}^{i r}$, the matrix $y=F^{e n}(P)$ is the only one in $\Phi(P)$ that can be written, for some strictly positive numbers $\lambda_{i}, \mu_{a}$, as

$$
y_{i a}=\lambda_{i} \mu_{a} * Q_{i a} \text { for all } i \in N, a \in A
$$

ii) Rationing: for any $P \in \mathcal{R}^{i r}$ with $x_{N}>r_{A}$, the matrix $y=H^{e n}(P)$ is the only one in $\Phi(P)$ that can be written, for some strictly positive numbers $z_{i}, \mu_{a}$, as

$$
\begin{gathered}
\qquad y_{i a}=z_{i} \mu_{a} * Q_{i a} \text { for all } i \in N, a \in A \\
\text { where for all } i: z_{i}=x_{i}-y_{i A} \Longleftrightarrow x_{i}=z_{i}+\sum_{A}\left(z_{i} \cdot \mu_{a}\right) * Q_{i a}
\end{gathered}
$$

For a possibly reducible problem, the statement is slightly less simple. Recall that $\tau(P)$ collects the entries $i a$ such that $y_{i a}$ is constant in $\Phi(P)$, while $\sigma(P)$ collects those such that $\left|Q_{i a}\right|=1$.

\section{Corollary 1}

i) For any $P \in \mathcal{A}$, the matrix $y=F^{e n}(P)$ is the only one in $\Phi(P)$ that can be written, for some strictly positive numbers $\lambda_{i}, \mu_{a}$, as

$$
y_{i a}=\lambda_{i} \mu_{a} * Q_{i a} \text { for all }(i, a) \notin \tau(P) \backslash \sigma(P)
$$


ii) For any $P \in \mathcal{R} \backslash \mathcal{A}$, the matrix $y=H^{e n}(P)$ is the only one in $\Phi(P)$ that can be written, for some strictly positive numbers $z_{i}, \mu_{a}$, as

$$
\begin{gathered}
y_{i a}=z_{i} \mu_{a} * Q_{i a} \text { for all }(i, a) \notin \tau(P) \backslash \sigma(P) \\
\text { where for all } i: z_{i}=x_{i}-y_{i A}
\end{gathered}
$$

In particular statement $i i)$ means that in a strictly rationed problem $\left(x_{N}>r_{A}\right)$ every agent incurs a positive deficit.

In the rationing example at the end of Section 3 the parametrization (7) of the capacited proportional matrix is $z_{1}=9 \frac{1}{3}, z_{2}=6 \frac{2}{3}, z_{3}=4$, and $\mu=\frac{5}{4}$.

Monotonicity properties of $F^{e n}$ and $H^{e n}$ To illustrate the power of the parametrization above, we deduce three natural monotonicity properties of the capacited proportional rules $F^{e n}$ and $H^{e n}$. The first property applies to both assignment and rationing rules $F, H$. We use the notation $Q_{[i]}$ for the $i$-th row of $Q$, and $Q^{[a]}$ for its $a$-column.

- Ranking for all $P \in \mathcal{R}$ all $i, j \in N:\left\{x_{i} \geq x_{j}\right.$ and $\left.Q_{[i]}=Q_{[j]}\right\} \Longrightarrow$ $y_{i a} \geq y_{j a}$ for all $a \in A$; for all $a, b \in A:\left\{r_{a} \geq r_{b}\right.$ and $\left.Q^{[a]}=Q^{[b]}\right\} \Longrightarrow$ $y_{i a} \geq y_{i b}$ for all $i \in N$.

The next two properties are for a rationing rule $H$. We compare two problems $P, P^{\prime} \in \mathcal{R}$ and the corresponding matrices $y, y^{\prime}$ :

- Sink Monotonicity: if $P=P^{\prime}$ except for $x_{i}<x_{i}^{\prime}$ then $y_{i A}<y_{i A}^{\prime}$ and $y_{j A} \geq y_{j A}^{\prime}$ for $j \neq i$.

- Source Monotonicity: if $P=P^{\prime}$ except for $r_{a}<r_{a}^{\prime}$ then $y_{i A} \leq y_{i A}^{\prime}$ for all $i$.

\section{Lemma 4}

i) Both rules $F^{e n}$ and $H^{e n}$ meet Ranking

ii) The rule $H^{e n}$ meets Sink and Source Monotonicity

\section{Characterization result and an open ques- tion}

\section{Theorem 1}

i) The assignment rule $F^{e n}$ is characterized, among all rules in $\mathcal{F}$, by the combination of three properties: it picks the proportional matrix in any unconstrained problem; Consistency; and Constraint Neutrality. 
ii) The rationing rule $H^{\text {en }}$ is characterized, among all rules in $\mathcal{H}$, by the combination of the same three properties.

Note that the two statements are not logically related. If $H \in \mathcal{H}$ meets the three properties, statement ii) implies that its restiction to $\mathcal{A}$ is $F^{e n}$. However statement $i$ ) characterizes $F^{e n}$ by means of axioms that only work in $\mathcal{F}$.

To see that statement $i$ ) is tight, pick any strictly convex function $W$ defined on $\mathbb{R}_{+}$and check that the rule $F(P)=\arg \min _{y \in \Phi(P)} \sum_{N \times A} W\left(y_{i a}\right)$ meets CSY and NEUT. Clearly for most choices of $W$ it does not pick the proportional assignment in an unconstrained problem $P \in \mathcal{A}^{u}$.

Next we discussed at the beginning of Section 4 a variety of functions $\mathcal{W}$ that are uniquely minimized by the proportional assignment in an unconstrained problem, yet the same minimization in $\mathcal{A}$ gives a rule failing CSY while meeting NEUT.

Finally the rule $F(P)=\arg \min _{y \in \Phi(P)} \sum_{N \times A} E n\left(\frac{y_{i a}}{1+q_{i a}^{-}}\right)$picks the proportional matrix when $Q=\mathbb{R}_{+}^{N \times A}$ and meets CSY, but fails NEUT.

We omit for brevity the similar proof that statement $i i$ ) is tight as well.

$A$ difficult open question. It is natural to generalize our model to allow for more general constraints on the matrix $y$ than the "rectangular" capacity constraints $Q$. For instance geographic constraints limit total number of some student types in two neighboring schools who share a bus system; see Budish et al. ([4]) for several more examples in the school choice context.

The natural domain contains all convex compact subsets of matrices. In Definition 3 we now mimimize the strictly convex total entropy over such a set, which is well defined. The corresponding rule meets the straightforward generalizations of axioms NEUT and CSY. It is unclear whether our characterization Theorem still holds in this more general domain.

\section{Appendix: proofs}

\subsection{Decomposition in irreducible problems}

Recall that $i a \in \tau(P)$ iff $y_{i a}$ is constant in $\Phi(P)$, and $i a \in \sigma(P)$ iff $q_{i a}^{-}=q_{i a}^{+}$. In an irreducible assignment problem $P \in \mathcal{A}^{i r}$ (Definition 1) the inclusion $\sigma(P) \subset \tau(P)$ may still be strict: consider for instance a column $a$ such that all entries $j a$ but $i a$ are in $\sigma(P)$ : then $y_{i a}=r_{a}-q_{(N \backslash i) a}^{-}$, so $i a \in \tau(P) \backslash \sigma(P){ }^{7}$ If we then remove column $a$, the reduced problem $P^{\prime}=\left(N, A \backslash\{a\}, x^{\prime}, r, Q\right)$ where $x_{i}^{\prime}=x_{i}-y_{i a}$, is still in $\mathcal{A}^{i r}$. Indeed if $P^{\prime}$ was reducible, we could

\footnotetext{
${ }^{7}$ By irreducibility we cannot have a full column or a full row contained in $\sigma(P)$.
} 
pick $S, T$, such that (2) is an equality, and check that (2) is an equality in $P$ for $S \cup\{i\}, T \cup\{a\}$, whether $i$ is in $S$ or not. A similar reduction by removing a row $i$ such that all entries $i b$ but $i a$ are in $\sigma(P)$ gives the irreducible assignment problem $P^{\prime}=\left(N \backslash\{i\}, A, x, r^{\prime}, Q\right)$ where $r_{a}^{\prime}=r_{a}-y_{i a}$. Repeating this reduction process until its end is useful in the proofs below.

For irreducible rationing problems $P \in \mathcal{R}^{i r} \backslash \mathcal{A}$, we can drop a column with just one entry outside $\sigma(P)$, but a row only if all entries are in $\sigma(P)$. The proof that this preserves irreducibility is omitted for brevity.

\section{Lemma 2}

i) For any $P \in \mathcal{A}^{\text {ir }}$ there is some feasible matrix $y \in \Phi(P)$ such that

$$
\text { for all } i a: q_{i a}^{-}<q_{i a}^{+} \Longrightarrow q_{i a}^{-}<y_{i a}<q_{i a}^{+}
$$

(recall $q_{i a}^{-}<q_{i a}^{+} \Leftrightarrow i a \notin \sigma(P)$ ). Moreover successive removals of columns or rows with all but one entry in $\sigma(P)$, leave us with a problem $P^{*} \in \mathcal{A}^{\text {ir }}$ that is either empty or has at least two entries outside $\sigma(P)$ in each row and in each column.

ii) For any $P \in \mathcal{R}^{i r} \backslash \mathcal{A}$ there is some feasible matrix $y \in \Phi(P)$ meeting (9) as well as

$$
\text { for all } i: y_{i A}<x_{i}
$$

Moreover successive removals of columns with all but one entry in $\sigma(P)$ and rows with all entries in $\sigma(P)$, leave us with a problem $P^{*} \in \mathcal{R}^{i r} \backslash \mathcal{A}$ that is either empty or has at least two entries outside $\sigma(P)$ in each column, and at least one in each row.

Proof To check (9) for $P$ in $\mathcal{A}^{i r}$, observe that for some small enough positive $\varepsilon$ we can change every constraint $Q_{i a}$ such that $q_{i a}^{-}<q_{i a}^{+}$into $Q_{i a}^{\prime}=$ $\left[q_{i a}^{-}+\varepsilon, q_{i a}^{+}-\varepsilon\right]$, and the resulting problem $\left(x, r ; Q^{\prime}\right)$ is still in $\mathcal{A}$. If $P \in \mathcal{R}^{i r} \backslash \mathcal{A}$, we change in addition $x_{i}$ to $x_{i}-\varepsilon$ and (10) follows

We note that the inclusion $\sigma\left(P^{*}\right) \subset \tau\left(P^{*}\right)$ may still be strict. An example is $N=\{1,2,3,4\}, A=\{a, b, c, d\}, x=(2,1,1,1), r=(2,1,1,1), Q_{1 b}=$ $Q_{2 a}=Q_{2 b}=Q_{i z}=\{0\}$ for $i=3,4$ and $z=c, d$, and $Q_{i z}=\mathbb{R}_{+}$otherwise. We have $P=P^{*} \in \mathcal{A}^{i r}$ and $y_{1 a}=1$ for all $y \in \Phi(P)$.

The next Lemma shows that any feasible problem can be decomposed into finitely many irreducible problems.

Lemma 3 (decomposition in irreducible problems) Each problem $P=$ $(N, A, x, r, Q)$ in $\mathcal{A}$ or $\mathcal{R}$ can be decomposed into irreducible subproblems $P^{k}$, $1 \leq k \leq K$, as follows. There are two partitions $N^{k}$ of $N$ and $A^{k}$ of $A$, $0 \leq k \leq K$, where $N^{0}$ and/or $A^{0}$ could be empty, such that

i) $\tau(P)$ contains each ia $\in N^{k} \times A^{k^{\prime}}$ with $k \neq k^{\prime}$ or $k=k^{\prime}=0$, and for such an entry we have $y_{i a}=\bar{q}_{i a} \in\left\{q_{i a}^{-}, q_{i a}^{+}\right\}$; 
ii) for all $k, 1 \leq k \leq K$, the subproblem $P^{k}=\left(N^{k}, A^{k}, x^{k}, r^{k} ; Q\right)$ where $x_{i}^{k}=x_{i}-\bar{q}_{i\left(A^{k}\right)^{c}}$ and $r_{a}^{k}=r_{a}-\bar{q}_{\left(N^{k}\right)^{c} a}$, is irreducible.

iii) if $P \in \mathcal{A}$, all subproblems $P^{k}$ are in $\mathcal{A}$ as well; if $P \in \mathcal{R}$, at most one subproblem $P^{k}$ is in $\mathcal{R}$, all others are in $\mathcal{A}$.

iv) if (2) is an equality for a pair $S, T, \varnothing \subseteq S \subseteq N, \varnothing \subseteq T \subseteq A$, then $S$ is a union of subsets $N_{k}$ and $T$ is the unions of the same subsets $A_{k}$.

Proof We prove statements $i$ ) to $i i i$ ) by induction on the dimension $|N|+|A|$. If $P$ is irreducible, we set $N^{1}=N, A^{1}=A$, and we are done. Assume now $P$ is reducible. Then there exist some subsets $S$ of $N, T$ of $A$, such that $r_{T}+q_{S T^{c}}^{-}=x_{S}+q_{S^{c} T}^{+}$, and we saw just before Definition 1 that this implies $y=q^{-}$on $S \times T^{c}$ and $y=q^{+}$on $S^{c} \times T$ for all $y \in \Phi(P)$.

If $S=\varnothing$ then $T \neq \varnothing$, so that $y=q^{+}$on $N \times T$ and the subproblem $\left(N, T^{c}, \widetilde{x}, r, Q\right)$ with $\widetilde{x}_{i}=x_{i}-q_{i T}^{+}$is clearly feasible. By induction we can decompose it. Note that $T$ will be a subset of $A^{0}$. Similarly if $S=N$ then $T \neq A$ and we get $y=q^{-}$on $N \times T^{c}$, so we decompose $(N, T, \widetilde{x}, r, Q)$ defined similarly; here $T^{c}$ ends up in $A^{0}$. The cases $T=\varnothing, S \neq \varnothing$, and $T=A, S \neq N$, give similarly $y=q^{-}$or $q^{+}$on $S \times A$ and $S^{c} \times A$ respectively, and $S^{c}$ or $S$ is a subset of $N^{0}$.

If $\varnothing \neq S \neq N$ and $\varnothing \neq T \neq A$ we have $y=q^{-}$on $S \times T^{c}$ and $q^{+}$on $S^{c} \times T$, which leaves us with two feasible subproblems: one balanced $(S, T, \widetilde{x}, \widetilde{r}, Q)$ with $\tilde{x}_{i}=x_{i}-q_{i T^{c}}^{-}, \widetilde{r}_{a}=r_{a}-q_{S^{c}}^{+}$; and the other $\left(S^{c}, T^{c}, \widehat{x}, \widehat{r}, Q\right)$ with $\widehat{x}_{i}=x_{i}-q_{i T}^{+}, \widehat{r}_{a}=r_{a}-q_{S a}^{-}$. If $P$ is an assignment problem, both subproblems are in $\mathcal{A}$; if $P$ is a strict rationing problem $\left(x_{N}>r_{A}\right)$, only subproblem $(S, T, \widetilde{x}, \widetilde{r}, Q)$ is balanced. The induction argument is now clear.

Statement $i v$ ) establishes uniqueness of the decomposition. We omit the proof for brevity, as it is not needed in subsequent proofs.

Lemma 3 implies that if two consistent rules $F, F^{\prime} \in \mathcal{F}$, or $H, H^{\prime} \in \mathcal{H}$, coincide on irreducible problems, they are the same rule.

\subsection{Parametrization of capacited proportionality}

We prove the Proposition in section 4. Combining this result and the canonical decomposition in Lemma 3 above gives the Corollary at once.

Statement i) Step 1 Fix $P \in \mathcal{A}^{i r}$. We show that $y=F^{e n}(P)$ can be written as (6). We use statement $i$ ) in Lemma 2 to first reduce $P$ to $P^{*}$ on $N^{*} \times A^{*}$ where every row and column has two or more entries outside $\sigma(P)$. Suppose we have shown (6) for $P^{*}=\left(N^{*} \times A^{*}, x^{*}, r^{*}, Q\right)$ where $x_{i}^{*}=x_{i}-y_{i\left(A^{*}\right)^{c}}$, and $r_{a}^{*}=r_{a}-y_{\left(N^{*}\right)^{c} a}$. Then we can choose $\lambda_{i}$ on $N$ and $\mu_{a}$ on $A$ sequentially to meet (6), starting from the last reduction just before reaching $P^{*}$. Say this last step eliminates row $i$ in which $i a$ is the only entry with a true interval 
$Q_{i a}$, and the only feasible value of $y_{i a}$ is $\bar{y}_{i a}$ : we know $\bar{y}_{i a}>0$ by statement $i$ ) in Lemma 2; also $\mu_{a}$ is determined in $P^{*}$ by assumption, and $\mu_{a}=0$ would imply $r_{a}^{*}=q_{N^{*} a}^{-}$which is excluded by irreducibility of $P^{*}$; therefore we can choose $\lambda_{i}>0$ to ensure $\bar{y}_{i a}=\lambda_{i} \mu_{a}$. If another row $j$ is removed at the last step, we can choose $\lambda_{j}$ as well. If a column $a$ is removed in the last step, and $i a$ is the only entry with a true interval $Q_{i a}$, we have similarly $\bar{y}_{i a}>0$ by statement $i$ ) in Lemma 2 , and $\lambda_{i}>0$ because $\lambda_{i}=0$ implies $x_{i}^{*}=q_{i A^{*}}^{-}$ contradicting irreducibility of $P^{*}$. Therefore we can choose $\mu_{a}>0$ to get $\bar{y}_{i a}=\lambda_{i} \mu_{a}$. And so on.

It remains to prove (6) when $y=F^{e n}(P)$ and each row and each column of $P$ has at least two entries outside $\sigma(P)$. Program (4) is equivalent to minimizing $\sum_{(N \times A) \backslash \sigma(P)}\left(E n\left(y_{i a}\right)-y_{i a}\right)$. We adjust the capacities of rows and columns accordingly by setting $x_{i}^{*}=x_{i}-\sum_{(i A) \cap \sigma(P)} y_{i a}$, and $r_{a}^{*}=r_{a}-$ $\sum_{(N a) \cap \sigma(P)} y_{i a}$. Then the program delivering $y=F^{e n}(P)$ has the following equality and inequality constraints:

$$
\begin{gathered}
y_{(i A) \backslash \sigma(P)}=x_{i}^{*} \text { for all } i, y_{(N a) \backslash \sigma(P)}=r_{a}^{*} \text { for all } a \\
y_{i a}-q_{i a}^{+} \leq 0, q_{i a}^{-}-y_{i a} \leq 0 \text { for } i a \in(N \times A) \backslash \sigma(P)
\end{gathered}
$$

The Lagrangian of the problem is $\mathcal{L}\left(y, \alpha, \beta, \theta^{+}, \theta^{-}\right)$, where $\alpha \in \mathbb{R}^{N}, \beta \in \mathbb{R}^{A}$ and $\theta^{+}, \theta^{-} \in \mathbb{R}_{+}^{(N \times A) \backslash \sigma(P)}$ :

$$
\begin{gathered}
\mathcal{L}\left(y, \alpha, \beta, \theta^{+}, \theta^{-}\right)=\sum_{(N \times A) \backslash \sigma(P)}\left(E n\left(y_{i a}\right)-y_{i a}\right)-\sum_{N} \alpha_{i}\left(y_{i A}-x_{i}\right)-\sum_{A} \beta_{a}\left(y_{N a}-r_{a}\right) \\
-\sum_{(N \times A) \backslash \sigma(P)} \theta_{i a}^{+}\left(q_{i a}^{+}-y_{i a}\right)-\sum_{(N \times A) \backslash \sigma(P)} \theta_{i a}^{-}\left(y_{i a}-q_{i a}^{-}\right)
\end{gathered}
$$

We check the qualification constraints. From $|(N \times A) \backslash \sigma(P)| \geq 2 \max \{|N|,|A|\}$ we see that the linear mapping $\mathbb{R}^{(N \times A) \backslash \sigma(P)} \ni y \rightarrow\left(y_{i A}, y_{N a}\right)_{i \in N, a \in A} \in \mathbb{R}^{N \cup A}$ is of maximal rank; and (9) says that there exists $y \in \Phi(P)$ such that $q_{i a}^{-}<y_{i a}<q_{i a}^{+}$for all $i a \in(N \times A) \backslash \sigma(P)$. Therefore there exist some KKT multipliers $\alpha, \beta, \theta^{+}, \theta^{-}$, such that

$$
\min _{y \in \Phi^{*}(P)} \sum_{(N \times A) \backslash \sigma(P)}\left(E n\left(y_{i a}\right)-y_{i a}\right)=\min _{y \in \mathbb{R}^{(N \times A) \backslash \sigma(P)}} \mathcal{L}\left(y, \alpha, \beta, \theta^{+}, \theta^{-}\right)
$$

where $\Phi^{*}(P)$ is the projection of $\Phi(P)$ on $\mathbb{R}^{(N \times A) \backslash \sigma(P)}$. Moreover $y=F^{e n}(P)$ if and only if $y$ minimizes the Lagrangian on the entire space, $y$ is feasible, and the complementarity properties $\theta_{i a}^{+}\left(y_{i a}-q_{i a}^{+}\right)=\theta_{i a}^{-}\left(q_{i a}^{-}-y_{i a}\right)=0$ hold for all $i a \in(N \times A) \backslash \sigma(P)$. 
The first order conditions are, for all $i a \in(N \times A) \backslash \sigma(P)$ :

$$
\ln \left(y_{i a}\right)=\alpha_{i}+\beta_{a}-\theta_{i a}^{+}+\theta_{i a}^{-}
$$

If $q_{i a}^{-}<y_{i a}<q_{i a}^{+}$, this reduces to $\ln \left(y_{i a}\right)=\alpha_{i}+\beta_{a} \Longleftrightarrow y_{i a}=e^{\alpha_{i}} e^{\beta_{a}}=$ $\left(e^{\alpha_{i}} e^{\beta_{a}}\right) * Q_{i a}$. If $y_{i a}=q_{i a}^{-}$we get $\ln \left(y_{i a}\right)=\alpha_{i}+\beta_{a}+\theta_{i a}^{-}$, implying $y_{i a} \geq e^{\alpha_{i}} e^{\beta_{a}}$ hence $y_{i a}=\left(e^{\alpha_{i}} e^{\beta_{a}}\right) * Q_{i a}$. Similarly $y_{i a}=q_{i a}^{+}$gives $y_{i a} \leq e^{\alpha_{i}} e^{\beta_{a}}=\left(e^{\alpha_{i}} e^{\beta_{a}}\right) *$ $Q_{i a}$. Thus (6) holds with $\lambda_{i}=e^{\alpha_{i}}, \mu_{a}=e^{\beta_{a}}$ for all $i a$ such that $Q_{i a}$ is not a singleton. And if $\left|Q_{i a}\right|=1$ this equality holds trivially. This concludes Step1.

Step 2. Conversely we take $y \in \Phi(P)$ and $\lambda_{i}, \mu_{a}$ meeting (6). We set $\alpha_{i}=\ln \left(\lambda_{i}\right), \beta_{a}=\ln \left(\mu_{a}\right)$, and

$\theta_{i a}^{+}=\theta_{i a}^{-}=0$ if $q_{i a}^{-}<y_{i a}<q_{i a}^{+}$

$\theta_{i a}^{+}=0$ and $\theta_{i a}^{-}=\ln \left(q_{i a}^{-}\right)-\alpha_{i}-\beta_{a}$ if $y_{i a}=q_{i a}^{-}$

$\theta_{i a}^{-}=0$ and $\theta_{i a}^{+}=-\ln \left(q_{i a}^{+}\right)+\alpha_{i}+\beta_{a}$ if $y_{i a}=q_{i a}^{+}$

It is then easy to check that the projection of $y$ on $\mathbb{R}^{(N \times A) \backslash \sigma(P)}$ minimizes $\mathcal{L}\left(y, \alpha, \beta, \theta^{+}, \theta^{-}\right)$in the entire space.

Statement ii) We sketch the parallel argument. For Step 1 we fix $P \in \mathcal{R}^{i r} \backslash \mathcal{A}$ and reduce it first to $P^{*}$ on $N^{*} \times A^{*}$ where each column has at least two entries outside $\sigma(P)$ and each row has at least one. Assuming (6) holds for $P^{*}$ we go backward from $P^{*}$ to $P$ as above. If the last step eliminated a row contained in $\sigma(P)$, then (7) holds for any choice of $z_{i}, \mu_{a}$, in particular for $\mu_{a}$ chosen for $P^{*}$ and for $z_{i}=x_{i}-y_{i A^{*}}$ which is positive by (10). If the last step eliminated a column with a single entry outside $\sigma(P)$, we mimick the argument in Step 1 taking into account $z_{i}>0$ for $i \in N^{*}$.

Working now with $P^{*}$ denoted simply $P$, program (5) is equivalent to minimizing $\sum_{(N \times A) \backslash \sigma(P)}\left(E n\left(y_{i a}\right)-y_{i a}\right)+\sum_{N} E n\left(x_{i}^{*}-y_{(i A) \backslash \sigma(P)}\right)$, with the following constraints:

$$
y_{(i A) \backslash \sigma(P)} \leq x_{i}^{*} \text { for all } i, y_{(N a) \backslash \sigma(P)}=r_{a}^{*} \text { for all } a \text {; and (11) }
$$

The new Lagrangian is $\mathcal{L}\left(y, \alpha, \beta, \theta^{+}, \theta^{-}\right)+\sum_{N} \operatorname{En}\left(x_{i}^{*}-y_{(i A) \backslash \sigma(P)}\right)$ where the parameter $\alpha$ is now in $\mathbb{R}_{-}^{N}$ instead of $\mathbb{R}^{N}$. The qualification constraints require, first, that the linear mapping $\mathbb{R}^{(N \times A) \backslash \sigma(P)} \ni y \rightarrow\left(y_{N a}\right)_{a \in A} \in \mathbb{R}^{A}$ be of maximal rank: this is clear from $|(N \times A) \backslash \sigma(P)| \geq|A|$ (in fact $\geq$ $2|A|)$. Second, properties (9) and (10) show there exists $y \in \Phi(P)$ where all inequality constraints are strict. Therefore there exist some KKT multipliers $\alpha, \beta, \theta^{+}, \theta^{-}$, such that

$$
\begin{aligned}
& \min _{y \in \Phi^{*}(P)} \sum_{(N \times A) \backslash \sigma(P)}\left(\operatorname{En}\left(y_{i a}\right)-y_{i a}\right)+\sum_{N} \operatorname{En}\left(x_{i}^{*}-y_{(i A) \backslash \sigma(P)}\right) \\
= & \min _{y \in \mathbb{R}^{(N \times A) \backslash \sigma(P)}} \mathcal{L}\left(y, \alpha, \beta, \theta^{+}, \theta^{-}\right)+\sum_{N} \operatorname{En}\left(x_{i}^{*}-y_{(i A) \backslash \sigma(P)}\right)
\end{aligned}
$$


Now $y=H^{e n}(P)$ if and only if $y$ minimizes the Lagrangian on the entire space, $y$ is feasible, and the complementarity properties $\alpha_{i}\left(y_{i A}-x_{i}\right)=$ $\theta_{i a}^{+}\left(y_{i a}-q_{i a}^{+}\right)=\theta_{i a}^{-}\left(q_{i a}^{-}-y_{i a}\right)=0$ hold for all $i a \in(N \times A) \backslash \sigma(P)$. The first order conditions are, for all $i a \in(N \times A) \backslash \sigma(P)$ :

$$
\ln \left(y_{i a}\right)-\ln \left(x_{i}^{*}-y_{(i A) \backslash \sigma(P)}\right)=\ln \left(y_{i a}\right)-\ln \left(x_{i}-y_{i A}\right)=\alpha_{i}+\beta_{a}-\theta_{i a}^{+}+\theta_{i a}^{-}
$$

As $\ln (0)$ is unbounded, we have $y_{i a}>0$ for all $i a \in(N \times A) \backslash \sigma(P)$ and $z_{i}=x_{i}-y_{i A}>0$ for all $i$, implying $\alpha=0$ and

$$
y_{i a}=z_{i} e^{\beta_{a}-\theta_{i a}^{+}+\theta_{i a}^{-}}
$$

As in the previous proof, this gives $y_{i a}=\left(z_{i} e^{\beta_{a}}\right) * Q_{i a}$ for all $i a \in(N \times$ $A) \backslash \sigma(P)$.

Step 2. Conversely we take $y \in \Phi(P)$ and $z_{i}, \mu_{a}$ meeting (7), (8). We have $z_{i}=x_{i}-y_{i A}=x_{i}^{*}-y_{(i A) \backslash \sigma(P)}$. We set $\alpha_{i}=0, \beta_{a}=\ln \left(\mu_{a}\right)$, and define $\theta^{+}, \theta^{-}$as above (with $\alpha=0$ ). The first order conditions (12) to minimize the Lagrangian are $\ln \left(y_{i a}\right)-\ln \left(y_{(i A) \backslash \sigma(P)}\right)=\beta_{a}-\theta_{i a}^{+}+\theta_{i a}^{-}$, and this holds true at $y$ by construction of $z, \beta, \theta^{ \pm}$.

\subsection{Lemma 4}

Statement $i$ ) If Ranking holds on every irreducible component of $P$ (Lemma 3 ), it clearly holds on $P$ itself. Thus we can assume $P \in \mathcal{R}^{i r}$ or $\mathcal{A}^{i r}$. For $P \in \mathcal{R}^{i r}$ we can assume $x_{i}>x_{j}$ (by Symmetry). If in system (7) we have $z_{i} \leq z_{j}$, then $y_{i A} \leq y_{j A} \Longleftrightarrow x_{i}-z_{i} \leq x_{j}-z_{j}$ and we reach a contradiction. Therefore $z_{i}>z_{j}$ and (7) gives $y_{i a} \geq y_{j a}$ for all $a$. The similar proofs for two columns, as well as for $P \in \mathcal{A}^{i r}$, are omitted for brevity.

Statement ii) For Sink Monotonicity, note that when we move from an arbitrary $P \in \mathcal{R}$ by only lifting sink 1 's capacity $x_{1}$, the irreducible decomposition in Lemma 3 changes only at finitely many points. Therefore by Continuity it is enough to prove the statement when the decomposition does not change from $x_{1}$ to $x_{1}^{\prime}$, and in turn this means we can assume $P$ and $P^{\prime}$ are both irreducible.

We set $z, \mu$ and $z^{\prime}, \mu^{\prime}$ for the corresponding parameters in (7), (8), and we note first $z_{N}<z_{N}^{\prime}$. We assume that the set $S=\left\{i \mid z_{i}>z_{i}^{\prime}\right\}$ is non empty and derive a contradiction. Indeed the set $T=\left\{a \mid \mu_{a}<\mu_{a}^{\prime}\right\}$ must be non empty, otherwise (7) implies $y_{i a} \geq y_{i a}^{\prime}$ for any $i$ in $S$ and any $a$; given $x_{i} \leq x_{i}^{\prime}$ this contradicts $z_{i}>z_{i}^{\prime}$. Observe now that $y_{i a} \leq y_{i a}^{\prime}$ holds on $S^{c} \times T$. As the capacity of sources does not change, this implies $y_{S T} \geq y_{S T}^{\prime}$. On the other hand we have $y_{i a} \geq y_{i a}^{\prime}$ on $S \times T^{c}$, and $z_{S}=x_{S}-y_{S A}>x_{S}^{\prime}-y_{S A}^{\prime}=z_{S}^{\prime}$. Together with $x_{S} \leq x_{S}^{\prime}$ this implies $y_{S T}^{\prime}>y_{S T}$, the desired contradiction. 
We have shown $z_{i} \leq z_{i}^{\prime}$ for all $i$. Hence $y_{i A} \geq y_{i A}^{\prime}$ for all $i \geq 2$, as was to be proved.

For Source Monotonicity, we can assume $P, P^{\prime} \in \mathcal{R}^{i r}$ for the same reason, and this time we have $z_{N}>z_{N}^{\prime}$. If the set $S=\left\{i \mid z_{i}<z_{i}^{\prime}\right\}$ is non empty, the set $T=\left\{a \mid \mu_{a}>\mu_{a}^{\prime}\right\}$ is non empty as well, by the same kind of argument as above. Similarly we have $y_{i a} \geq y_{i a}^{\prime}$ on $S^{c} \times T$, hence $y_{S T} \leq y_{S T}^{\prime}$; on the other hand $y_{i a} \leq y_{i a}^{\prime}$ on $S \times T^{c}$, together with $z_{S}=x_{S}-y_{S A}<x_{S}-y_{S A}^{\prime}=z_{S}^{\prime}$ implies $y_{S T}^{\prime}>y_{S T}$, contradiction. We have shown $z_{i} \geq z_{i}^{\prime}$ for all $i$. Hence $z_{i}=x_{i}-y_{i A} \geq x_{i}-y_{i A}^{\prime}=z_{i}^{\prime}$ for all $i$ and we are done.

\subsection{Characterization result}

We prove statement $i$ ) of the Theorem in steps 1 and 2 . We already know that $H^{e n}$ meets the three properties. So we pick $H \in \mathcal{H}$ meeting them as well and we must show $H=H^{e n}$. By Lemma 3 it is enough to show $H(P)=H^{e n}(P)$ for $P \in \mathcal{R}^{i r}$.

Step 1 We prove this first for standard rationing problems, namely when $|A|=1$. As a reminder we write such a problem as $P^{s}$. All rules coincide on $P^{s} \in \mathcal{A}$, thus we only need to consider $P^{s} \in \mathcal{R}^{i r} \backslash \mathcal{A}$. By Symmetry in $A$, the choice of $A$ does not matter so we can write a standard problem as $P^{s}=(N, x, t, Q)$ where $t \geq 0$ is the amount to share and $Q_{i}=\left[q_{i}^{-}, q_{i}^{+}\right]$for all $i$. Irreducibility of $P^{s}$ means

$$
q_{N}^{-}<t<q_{N}^{+} \text {and for all } S, \varnothing \neq S \neq N: q_{S}^{-}<x_{S} \text { and } t<x_{S}+q_{S^{c}}^{+}
$$

We show that $H\left(P^{s}\right)=y$ takes the form $y_{i}=\mu z_{i} * Q_{i}$ for all $i$, where $\mu>0$ and $z_{i}=x_{i}-y_{i}>0$. The proof is by induction on the number $v$ of strict inequalities $q_{i}^{-}>0$ and $q_{i}^{+}<\infty$. If $v=0$ the rule $H$ is proportional by assumption. Fix now a problem $P^{s}$ with $v+1$ strict inequalities, and single out one of them for instance $q_{1}^{+}<\infty$. We let $Q^{\prime}$ be the same as $Q$ except that $Q_{1}^{\prime}=\left[q_{1}^{-}, \infty\left[\right.\right.$, so the problem $P^{s \prime}=\left(N, x, t, Q^{\prime}\right)$ is still irreducible. By the inductive assumption $H\left(P^{s \prime}\right)=H^{e n}\left(P^{s \prime}\right)$ and we call this allocation $y^{\prime}$.

If $y_{1}^{\prime} \leq q_{1}^{+}$NEUT $($part $i)$ ) implies $H\left(P^{s \prime}\right)=H\left(P^{s}\right)$ and $H^{e n}\left(P^{s \prime}\right)=$ $H^{e n}\left(P^{s}\right)$, so we are done. Assume now $y_{1}^{\prime}>q_{1}^{+}$and note that $y_{1}<q_{1}^{+}$would imply (by NEUT part $i i$ ) $y=y^{\prime}$, a contradiction. Thus $y_{1}=q_{1}^{+}$.

We use the notation $y_{-1}$ for the projection of $y$ on $\mathbb{R}_{+}^{N \backslash 1}$, and $P^{s}[-1]=$ $\left(N \backslash 1, x_{-1}, t-q_{1}^{+}, Q\right)$, still an irreducible problem. CSY applied to the removal of sink 1 in $H$ and $H^{e n}$ implies $y_{-1}=H\left(P^{s}[-1]\right)$ and $y_{-1}=$ $H^{e n}\left(P^{s}[-1]\right)$. The inductive assumption says there exists $\mu>0$ such that $y_{i}=\mu\left(x_{i}-y_{i}\right) * Q_{i}$ and $x_{i}>y_{i}$ for all $i \geq 2$. On the other hand $y^{\prime}=$ $H^{e n}\left(P^{s \prime}\right)$ so the Proposition in Section 4 shows there exists $\lambda>0$ such that 
$y_{i}^{\prime}=\lambda\left(x_{i}-y_{i}^{\prime}\right) * Q_{i}$ and $x_{i}>y_{i}^{\prime}$ for all $i \geq 2$, and $y_{1}^{\prime}=\lambda\left(x_{1}-y_{1}^{\prime}\right) *\left[q_{1}^{-}, \infty[\right.$. As $y_{1}^{\prime}>q_{1}^{+}$the latter equality gives $y_{1}^{\prime}=\lambda\left(x_{1}-y_{1}^{\prime}\right)=\frac{\lambda}{\lambda+1} x_{1}$.

Next it is easy to check the following equivalence

$$
y_{i}=\mu\left(x_{i}-y_{i}\right) * Q_{i} \Longleftrightarrow y_{i}=\frac{\mu}{\mu+1} x_{i} * Q_{i} \text { for all } i \geq 2
$$

(for instance from left to right, $\mu\left(x_{i}-y_{i}\right) \geq q_{i}^{+} \Longrightarrow y_{i}=q_{i}^{+} \Longrightarrow \frac{\mu}{\mu+1} x_{i} \geq q_{i}^{+}$, etc.). Similarly $y_{i}^{\prime}=\frac{\lambda}{\lambda+1} x_{i} * Q_{i}$ for all $i \geq 2$. From $y_{N \backslash 1}=t-q_{1}^{+}>y_{N \backslash 1}^{\prime}$ we have $\mu>\lambda$ hence $\frac{\mu}{\mu+1} x_{1}>\frac{\lambda}{\lambda+1} x_{1}>q_{1}^{+}$and finally $y_{1}=q_{1}^{+}=\mu\left(x_{1}-y_{1}\right) * Q_{1}$. Thus $y=H\left(P^{s}\right)$ takes the parametric form (7),(8), concluding the induction step when we single out a non trivial upper bound constraint $q_{1}^{+}<\infty$. We omit the similar proof when we pick a non trivial lower bound.

Step 2 We take now a problem $P \in \mathcal{R}^{i r} \backslash \mathcal{A}$ where $|A|$ is arbitrary, and we set $y=H(P)$ with a deficit vector $z_{i}=x_{i}-y_{i A}$. By assumption $z_{N}>0$. Removing all sources but $a$ gives a reduced capacity $x_{i}-y_{i(A \backslash a)}=z_{i}+y_{i a}$ for sink $i$. With the notation $y^{[a]}$ for the $a$-column of $y$ and similarly for $Q$, CSY and step 1 imply

$$
y^{[a]}=H\left(N, z+y^{[a]}, r_{a}, Q^{[a]}\right)=H^{e n}\left(N, z+y^{[a]}, r_{a}, Q^{[a]}\right)
$$

Suppose $z_{i}>0$ for all $i$. Then each standard problem $P^{a}=(N, z+$ $\left.y^{[a]}, r_{a}, Q^{[a]}\right)$ is irreducible: $q_{N a}^{-}<r_{a}<q_{N a}^{+}$follows from $P \in \mathcal{R}^{i r}$, and for $S \neq \varnothing, N$, we have $q_{S a}^{-} \leq y_{S a}<z_{S}+y_{S a}$ and $r_{a} \leq y_{S a}+q_{S^{c} a}^{+}<z_{S}+y_{S a}+q_{S^{c} a}^{+}$. Therefore step 1 gives for each $a$ a positive $\mu_{a}$ such that $y_{i a}=\mu_{a} z_{i} * Q_{i a}$ for all $i$ and we are done.

We now assume that $S^{*}=\left\{i \in N \mid z_{i}=0\right\}$ is non empty, and we derive a contradiction. Note that $\left(S^{*}\right)^{c}$ is non empty as well $\left(z_{N}>0\right)$, so the problem $P^{a}$ is in $\mathcal{R} \backslash \mathcal{A}$ for each $a$. It cannot be irreducible: by statement $\left.i i\right)$ in the Proposition of Section 4 such a problem has $z_{i}>0$ for all $i$. Thus for each $a$ system (13) is violated: there is some $S, \varnothing \neq S \neq N$, such that at least one of

$$
r_{a}=z_{S}+y_{S a}+q_{S^{c} a}^{+}
$$

and/or

$$
q_{S a}^{-}=z_{S}+y_{S a}
$$

holds. Let $B$ be the possibly empty subset of sources $a$ such that $y_{i a}=q_{i a}^{+}$ for all $i \in\left(S^{*}\right)^{c}$. We claim that $y_{i a}=q_{i a}^{-}$for $i \in S^{*}$ and $a \in B^{c}$.

Indeed if (14) holds at $a$, we deduce from $r_{a}=y_{S^{c} a}+y_{S a}$ that $z_{S}=0$ and $y_{i a}=q_{i a}^{+}$for all $i \in S^{c}$, i. e., $a \in B$. Fixing any $a$ outside $B$, equation (15) holds for some $S$. This implies $z_{S}=0 \Rightarrow S \subseteq S^{*}$, and $y_{i a}=q_{i a}^{-}$for all $i \in S$. If $S=S^{*}$ the claim is proven at $a$, so we assume $S \varsubsetneqq S^{*}$. Consider the 
reduction $\widetilde{P}^{a}$ of $P^{a}$ to $S^{c}$, where these sinks share $\widetilde{r}_{a}=r_{a}-y_{S a}$ : by CSY in $\widetilde{P}^{a}$ sink $i$ still gets $y_{i a}$ and his deficit is still $z_{i}$. Therefore $\widetilde{P}^{a}$ is reducible $\left(S^{c}\right.$ contains some $i \in S^{*}$ where $\left.z_{i}=0\right)$ so there is some $R, \varnothing \neq R \neq S^{c}$, such that (14) and/or (15) holds. But (14) is $\widetilde{r}_{a}=z_{R}+y_{R a}+q_{(S \cup R)^{c} a}^{+} \Longleftrightarrow r_{a}=$ $z_{S \cup R}+y_{(S \cup R) a}+q_{(S \cup R)^{c} a}^{+}$, which implies $y_{i a}=q_{i a}^{+}$on $(S \cup R)^{c} \times\{a\} \supseteq S^{*}$, and we have excluded this possibility outside $B$. Thus (15) holds, implying as above $z_{R}=0$ and $y_{i a}=q_{i a}^{-}$on $R$. Thus $R \subset S^{*}$ and the desired equality $y_{i a}=q_{i a}^{-}$holds on $S \cup R$ ( $a$ is still fixed). Repeating the argument we reach the claim.

We have shown that $y_{i a}=q_{i a}^{+}$on $\left(S^{*}\right)^{c} \times B$, while $y_{i a}=q_{i a}^{-}$on $S^{*} \times B^{c}$. Then the accounting identity $r_{B}+y_{S^{*} B^{c}}=x_{S^{*}}+y_{\left(S^{*}\right)^{c} B}$ implies $r_{B}+q_{S^{*} B^{c}}^{-}=$ $x_{S^{*}}+q_{\left(S^{*}\right)^{c} B}^{+}$, in contradiction of the irreducibility of $P$.

Step 3 We prove statement $i$ ). Pick $F \in \mathcal{F}$ meeting the three properties and construct a standard rationing rule denoted $h$ as follows. The small letter reminds us that this rule is only defined for problems with a single source. For each such problem $P^{s}=(N, x, t, Q)$ we construct an assignment problem $P=(N,\{a, b\}, x, r, \widetilde{Q})$ where $r_{a}=t, r_{b}=x_{N}-t$, and $\widetilde{Q}^{[a]}=Q, \widetilde{Q}^{[b]}=\mathbb{R}_{+}^{N}$. Then we define $h\left(P^{s}\right)$ to be the $a$-column of $F(P)$.

By Symmetry in $A$ the choice of $a, b$, does not matter. It is equally clear that the standard rationing rule $P^{s} \rightarrow h\left(P^{s}\right)$ is symmetric in $N$, continuous, and satisfies NEUT and CSY because $F$ does. Therefore by step 1 we have $h\left(P^{s}\right)=h^{e n}\left(P^{s}\right)$.

We pick now $P=(N, A, x, r, Q) \in \mathcal{A}^{i r}$ and we show $F(P)=F^{e n}(P)$. By Lemma 3 this is all we need to prove. We construct for all $\varepsilon \geq 0$ an augmented problem $P[\varepsilon]=\left(N, A \cup\left\{a^{*}\right\}, x^{\varepsilon}, r^{\varepsilon}, Q^{\varepsilon}\right)$ with one more source $a^{*}$ as follows:

$$
x_{i}^{\varepsilon}=x_{i}+\frac{\varepsilon}{|N|} \text { for all } i ; r^{\varepsilon}=(r, \varepsilon) ; Q_{i a^{*}}^{\varepsilon}=\mathbb{R}_{+}, Q_{i a}^{\varepsilon}=Q_{i a} \text { otherwise }
$$

It is easy to check that $P[\varepsilon]$ is in $\mathcal{A}^{i r}$ as well. Set $y=F(P)$ and $\left(y^{\varepsilon}, z^{\varepsilon}\right)=$ $F(P[\varepsilon])$ where $z_{i}^{\varepsilon}$ is the $i a^{*}$ coordinate of $F(P[\varepsilon])$ and $y^{\varepsilon}$ is of dimension $N \times A$. By Continuity we have $\lim _{\varepsilon \rightarrow 0}\left(y^{\varepsilon}, z^{\varepsilon}\right)=F(P[0])$ and by CSY $F(P[0])=$ $(y, 0)$. The same remarks apply to $F^{e n}(P)$ and $F^{e n}(P[\varepsilon])$, therefore it is enough to prove $F(P[\varepsilon])=F^{e n}(P[\varepsilon])$ for all $\varepsilon>0$. For simplicity we write now $y, z$ instead of $y^{\varepsilon}, z^{\varepsilon}$.

For any $a \in A$ we reduce $P[\varepsilon]$ to a problem in $\mathcal{A}$ on $N \times\left\{a, a^{*}\right\}$ by dropping all other sources. Sink $i$ 's reduced capacity is $x_{i}-y_{i(A \backslash a)}=z_{i}+y_{i a}$. Setting $\widetilde{Q}_{i a}=Q_{i a}$ and $\widetilde{Q}_{i a^{*}}=\mathbb{R}_{+}$and applying CSY we have

$$
\left(y^{[a]}, z\right)=F\left(N,\left\{a, a^{*}\right\}, z+y^{[a]},\left(r_{a}, \varepsilon\right), \widetilde{Q}\right) \Longrightarrow y^{[a]}=h\left(N, z+y^{[a]}, r_{a}, Q^{[a]}\right)
$$


The proof now follows exactly the pattern of step 2. If $z_{i}>0$ for all $i$, each standard problem $P^{a}=\left(N, z+y^{[a]}, r_{a}, Q^{[a]}\right)$ is irreducible. From $h=h^{e n}$ we deduce, as in step 2, $y_{i a}=\mu_{a} z_{i} * Q_{i a}$ for some $\mu_{a}>0$ and all $i$. In addition we choose $\mu_{a^{*}}=1$ so that $(y, z)$ has the parametric form (6) everywhere and this gives $F(P[\varepsilon])=F^{e n}(P[\varepsilon])$ by statement $\left.i\right)$ in the Proposition.

If $S^{*}=\left\{i \in N \mid z_{i}=0\right\}$ and $\left(S^{*}\right)^{c}$ are non empty, $P^{a}$ is in $\mathcal{R} \backslash \mathcal{A}$ for all $a$ and is not irreducible for the same reasons. Thus (13) is violated for each $a$ : there is some $S, \varnothing \neq S \neq N$, such that $r_{a}=z_{S}+y_{S a}+q_{S^{c} a}^{+}$and/or $q_{S a}^{-}=z_{S}+y_{S a}$. As in the previous step this yields a partition $\left\{S^{*},\left(S^{*}\right)^{c}\right\}$ of $N$ (both sets non empty) and $\left(B, B^{c}\right)$ of $A$ (at least one non empty) such that $r_{B}+q_{S^{*} B^{c}}^{-}=x_{S^{*}}+q_{\left(S^{*}\right)^{c} B}^{+}$, in contradiction of the irreducibility of $P$.

\section{References}

[1] M.L. Balinski and G. Demange, 1989, An axiomatic approach to proportionality between matrices, Mathematics of Operations Research, 14, $4,700-719$.

[2] Balinski, M., and H.P. Young, 1982, Fair Representation:Meeting the Ideal of One Man one Vote, Yale University Press.

[3] R.D. Banker, 1981, Equity Considerations in Traditional Full Cost AIlocation Practices: An Axiomatic Perspective, in Joint Cost Allocations, S. Moriarty EDs, Norman: University of Oklahoma.

[4] Budish, E., Che, YK., Kojima, F., and P. Milgrom, 2013, Designing Random Allocation Mechanisms: Theory and Applications, American Economic Review, 103, 2, p. 585-623.

[5] G. Bergantinos and F. Sanchez, 2002, The proportional rule for problems with constraints and claims, Mathematical Social Sciences, 43, 2, 225249.

[6] O. Bochet, R. Ilkilic, and H. Moulin, 2013, Egalitarianism under Earmark Constraints, Journal of Economic Theory, 148, 535-562.

[7] O. Bochet, R. Ilkilic, H. Moulin, and J. Sethuraman, 2012, Balancing Supply and Demand under Bilateral Constraints, Theoretical Economics, 7,3, 395-424.

[8] C. Chambers, 2004, Consistency in the probabilistic assignment model, Journal of Mathematical Economics, 40, 953-962 
[9] E. Engle, 2012, The History of the General Principle of Proportionality: An Overview, Dartmouth Law Journal, 10, 1-11.

[10] Y. Flückiger, J. Silber, 1999, The Measurement of Segregation in the Labor Force, Physica-Verlag, Heidelberg and New York.

[11] D. Frankel, O. Volij, 2011, Measuring school segregation, Journal of Economic Theory, 146, 1-38.

[12] J. L. Hougaard, J. D. Moreno Ternero, L. P. Osterdal, 2012, A unifying framework for the problem of adjudicating conflicting claims, Journal of Mathematical Economics, 48, 2, March 2012, 107-114.

[13] R. Hutchens, 2001, Numerical measures of segregation: desirable properties and their implications, Mathematical Social Sciences 42, 13-29.

[14] M. Kaminski, Hydraulic rationing, Mathematical Social Sciences 40, 2, $131-155$.

[15] H. Moulin, 20003, Fair Division and Collective Welfare, MIT Press, Cambridge: Mass.

[16] H. Moulin, 2014, Consistent bilateral assignment and rationing, University of Glasgow, http://www.gla.ac.uk/media/media_382976_en.pdf.

[17] H. Moulin and J. Sethuraman, 2013a, The bipartite rationing problem, Operations Research, 61 (5). pp. 1087-1100.

[18] H. Moulin and J. Sethuraman, 2013b, Loss calibrated rationing methods for bipartite rationing, (with Jay Sethuraman), Proceedings of the 14th Electronic Commerce Conference (EC13), June 20-24, 2013, Philadelphia, Pennsylvania.

[19] A. Sen, 1996, On the Status of Equality, Political Theory, 24, 3, 394-400.

[20] H. Theil, A.J. Finizza, 1971, A note on the measurement of racial integration in schools, J. Mathematical Sociology 1, 187-193.

[21] W. Thomson, 2003, Axiomatic and game-theoretic analysis of bankruptcy and taxation problems", Mathematical Social Sciences, 45, 3, 249-297.

[22] W. Thomson, 2011, Consistency and its converse: an introduction, Review of Economic Design 15 (4), 257-291. 
[23] W. Thomson, 2012, On the axiomatics of resource allocation: Interpreting the consistency principle, Economics and Philosophy 28 (3), 385-421.

[24] H. P. Young, 1987, "On dividing an amount according to individual claims or liabilities," Mathematics of Operations Research, 12, 397-414.

[25] H. P. Young, 1994, Equity: in Theory and practice, Princeton University Press. 